\title{
Proceedings of the 10th Workshop on Embedded Systems Security
}

A Workshop of the Embedded Systems Week 2015, Amsterdam, The Netherlands

WESS 2015

\author{
Technical Program Chairs \\ Stavros Koubias (University of Patras) \\ Thilo Sauter (Donau University Krems)
}

\author{
Steering Committee \\ Catherine Gebotys (University of Waterloo, Canada) \\ Dimitrios Serpanos (Qatar Computing Research Institute) \\ Marilyn Wolf (Georgia Tech, US)
}




\section{Table of Contents}

1. Towards Continuous Authentication in Internet of Things Based on Secret Sharing Scheme

Omaimah Bamasag and Kamal Youcef-Toumi

2. A Novel Approach to Detect Hardware Trojan Attacks on Primary Data Inputs Taimour Wehbe, Vincent J. Mooney, David C. Keezer and Nicholas B. Parham

3. Analysis of Control Flow Events for Timing-based Runtime Anomaly Detection Sixing Lu and Roman Lysecky

4. Bitslice software implementation of KeeLoq as a side-channel countermeasure Pedro Malagón, Juan-Mariano De Goyeneche, David Fraga and Jose M. Moya

5. Testing Feasibility of Back-Side Laser Fault Injection on a Microcontroller Jakub Breier and Dirmanto Jap

6. A Novel Attack on a FPGA based True Random Number Generator Anju Johnson, Rajat Chakraborty and Debdeep Mukhopadhyay

7. Performance analysis of broadcast authentication protocols on CAN-FD and FlexRay Paula Vasile, Bogdan Groza and Pal-Stefan Murvay

8. Abstract Data Machine: Data Classifier for Reliable Embedded Systems Software Muhammad Taimoor Khan, Anastasios Fragopoulos, Dimitrios Serpanos and Howard Shrobe

9. CHEF: A Configurable Hardware Trojan Evaluation Framework Christian Krieg and Daniel Neubacher

10. Security Challenges in ManyCore Embedded Systems based on Networks-on-Chip Ermis Papastefanakis, Dominique Ragot and Bruno Maitre 